\title{
Evaluation of Wind Energy Accommodation Based on Two-Stage Robust Optimization
}

\author{
Kunpeng Tian ${ }^{1}$, Weiqing Sun ${ }^{2,}$, Dong $\mathrm{Han}^{2}$, Ce Yang ${ }^{1}$ \\ ${ }^{I}$ Department of Control Science and Engineering, University of Shanghai for Science and Technology, \\ Shanghai 200093, China \\ ${ }^{2}$ Department of Electrical Engineering, University of Shanghai for Science and Technology, \\ Shanghai 200093, China \\ sunwq@usst.edu.cn
}

\begin{abstract}
Large-scale renewable energy integration brings unprecedented challenges to electric power system planning and operation. The paper aims at economic dispatch and the safe operation of high penetration renewable energy power systems. According to the principle of power system dispatchability, the assessment of wind energy accommodation is formulated into a two-stage robust optimization problem with a min-max-min structure. Based on the benders algorithm, the intractable robust optimization problem is transformed into the form of sub-problem and master problem. Strong duality theory and big-M method are used to recast the sub problem into a mixed integer linear programming. The envelope of wind energy accommodation can be obtained by using commercial software to solve the master problem and sub problem alternately. For the realization of arbitrary wind power within the envelope, the amount of wind energy leakage and load shedding in power system operation are acceptable. An example of modified IEEE 39-bus test systems is used to verify the effectiveness and practicability of the evaluation method.
\end{abstract}

Index Terms-Economic dispatch; Wind energy accommodation; Two-stage robust optimization; Benders algorithm.

\section{INTRODUCTION}

Wind power with economic and environmental benefits has been integrated into the large electric power system, which will endanger the security and stability of the power industry [1]. In recent years, the high penetration of renewable energy (wind energy and solar energy) has become the development trend of the modern energy system, which acts a pivotal part in the electric power industry of all countries in the world. Take wind power for example, the installed capacity of wind turbine (WT) has increased about 20 times from $24 \mathrm{GW}$ in 2001 to $487 \mathrm{GW}$ in 2016 [2]. Although large-scale wind farms have been integrated into the energy system [3], the proportion of wind energy in the terminal energy structure has not reached the expected level because the production power of WT depends on the natural environment. China's installed capacity of WT ranks first in the world. However, the problem of wind power spillage has become increasingly prominent in

Manuscript received 16 January, 2020; accepted 28 April, 2020.

This research was funded by the Natural Science Foundation of China under Grant (No. 51777126). recent years. The National Energy Agency statistics show that the national average rate of wind power spillage was about $20 \%$ in 2017 [4]. Accurate and effective evaluation of wind energy accommodation can improve the utilization rate of electric power equipment, which is of great significance to improve the economy and security of electric energy system. The problem of wind energy accommodation has attracted the attention of scholars all over the world. The evaluation and calculation of wind energy accommodation is a complex problem considering the factors of the generator, transmission lines, and load demand in the regional power system [5]. The existing research on assessment of wind power accommodation uses different modeling techniques to study the accommodation problem from different aspects.

From the perspective of definitions and concepts, flexibility is described as the capability of energy system to counterweight net load demand fluctuations by adjusting the operating state of controllable resources [6]. However, the capability to handle expected and unexpected events is limited by several security constraints. Reference [7] proposes a novel framework for the definition and evaluation of power system flexibility based on the four dimensions. The largest accommodate range of variation is used to evaluate the flexibility, which is obtained by solving an optimization model considering the economic and security constraints of the electric energy system. Reference [8] reviews the concepts and indices of electrical power systems flexibility, discuss the relationship between reserve capacity and economy in electric power system and analyzes the impact of renewable energy penetration on flexibility. Reference [9] proposes the concept of a flexible envelope to handle the uncertainty and variability in the power balance of high penetration renewable energy system. Reference [10] emphasizes that power system dispatchability is the key to cope with the variability of renewable energy production.

From the perspective of methodological and model, an energy reserve joint optimal dispatch model for wind energy accommodation is proposed based on two indicators: loss of load probability and wind power spillover probability [11]. Reference [12] establishes an accurate multi-period AC model to optimize the installed capacity of the distributed generation. Reference [13] proposes a renewable energy 
accommodation model, which is the sequential production simulation model and considering the stability constraints of the power system and transmission line constraints. Reference [14] puts forward an optimization model with the Do-Not-Exceed (DNE) limit as the objective to evaluate the maximum renewable energy generation interval that the power grid can accommodate without sacrificing the system safety indicator. Based on the DNE limits, a real-time scheduling method of hybrid robust optimization and stochastic optimization is proposed to improve the level of renewable energy accommodation [15].

The power system has the characteristics of a real-time balance between production and demand. Therefore, the concept of flexibility can be applied to assess the capacity of renewable energy accommodation. Similar to flexibility, the ability of accommodate capacity is the natural characteristic of energy system, which has the ability to accommodate wind energy and photovoltaic. The difference between flexibility and wind power accommodation assessment is that the former focuses on the ability of the system to deal with the uncertainty of net load, while the latter emphasizes the ability of the system to deal with the uncertainty of wind production power. Although a large number of reports have analyzed the problem of renewable energy accommodation assessment, there is insufficient research on the unified evaluation framework, which takes into account the power supply, transmission lines, load, and other factors.

This paper aims at the dynamic economic dispatch of electric energy system with wind energy and proposes a novel evaluation framework of wind energy accommodation. This framework integrates the day-ahead unit commitment and real-time economic dispatching into the evaluation model of wind energy accommodation and provides the wind energy envelope, and which can guide the real-time operation of power system. The main works of this paper are as follows:

1. Based on the theory of pre-dispatch and re-dispatch in the power system, the relationship between dispatchability and wind energy accommodation is analyzed;

2. Based on the dynamic economic dispatch model and two-stage robust optimization technology, an assessment framework of wind energy accommodation taking into wind energy leakage and load demand shedding is proposed;

3. The framework provides a deterministic envelopment metrics that can be used as a decision-making tool to provide theoretical support for the economic operation of power grid.

The rest of this paper is organized as follows. The concept of power system dispatchability is described in Section II. The evaluation framework of wind energy accommodation is proposed in Section III. The solution methodologies are presented in Section IV. Numerical studies are conducted and simulation results are analyzed in Section V. Finally, the conclusions are summarized in Section VI.

\section{POWER SYSTEMS DiSPATCHABILITY}

In this section, based on the theory of electric power system pre-dispatch and re-dispatch [16], the concept of system dispatchability is proposed. First, the security-constrained unit commitment (SCUC) problem is solved to determine the start-up and shut-down of the thermal generator (TG) [17]. Second, the assessment of wind power accommodation is solved to obtain the largest operating ranges of each WT. We take a simple system that includes two generators as an example to illustrate the process of power system pre-dispatch and re-dispatch as follows in Fig. 1.

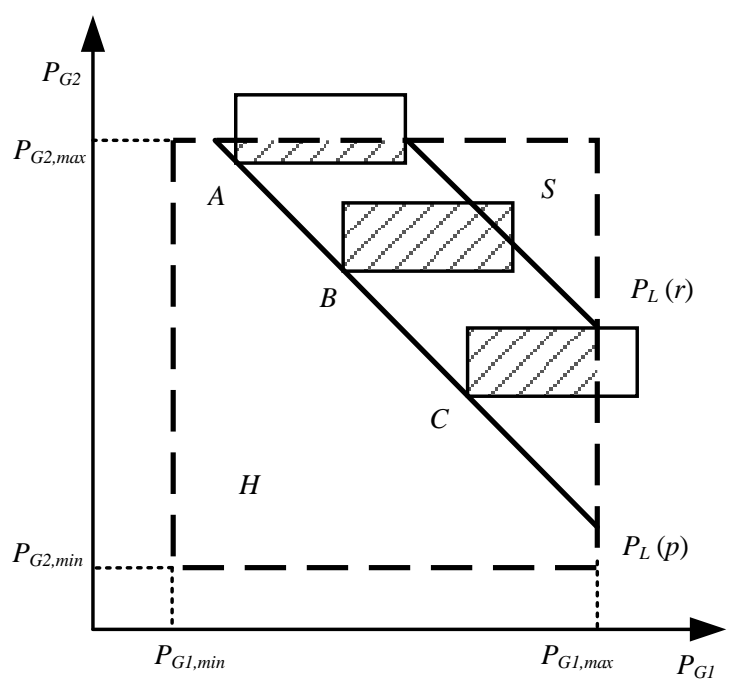

Fig. 1. Power system pre-dispatch and re-dispatch process.

The coordinate axis in Fig. 1 represents the out power of the generator. $P_{G 1, \min }$ and $P_{G 1, \max }$ represent the lower and upper bounds of production capacity, respectively. Rectangle $H$ indicates the feasible region of the production power of two generators. $P_{L}(p)$ and $P_{L}(r)$ are the day-ahead and real-time load demand, respectively. Then, $L(p)$ and $L(r)$ are day-ahead and real-time dispatch schemes, respectively:

$$
\begin{aligned}
& L(p)=\left\{\left(P_{G 1}^{(p)}, P_{G 2}^{(p)}\right) \mid P_{G 1}^{(p)}+P_{G 2}^{(p)}=P_{L}^{(p)}\right\}, \\
& L(r)=\left\{\left(P_{G 1}^{(r)}, P_{G 2}^{(r)}\right) \mid P_{G 1}^{(r)}+P_{G 2}^{(r)}=P_{L}^{(r)}\right\},
\end{aligned}
$$

where $P_{G 1}^{(p)}$ and $P_{G 2}^{(p)}$ are the pre-dispatch production power of TG in day-ahead stage and $P_{G 1}^{(r)}$ and $P_{G 2}^{(r)}$ are the re-dispatch production power of TG in real-time stage. From the perspective of ramp, the relationship between of pre-dispatch scheme and re-dispatch scheme is shown as follows

$$
L(r)=\left\{\left(P_{G 1}^{(p)}, P_{G 2}^{(p)}\right) \mid P_{G 1}^{(p)}+R_{1}^{u} \Delta t+P_{G 2}^{(p)}+R_{2}^{u} \Delta t=P_{L}^{(r)}\right\}
$$

where $R_{1}^{u}$ and $R_{2}^{u}$ are the ramping capacity of TG, and $\Delta t$ is the allowable adjustment time. Assuming that the prediction error is positive and this constraint needs to be satisfied to avoid load curtailment

$$
R_{1}^{u} \Delta t+R_{2}^{u} \Delta t \geq P_{L}^{(r)}-P_{L}^{(d)}
$$

The shadow rectangle $S$ in Fig. 1 represents the ramping capacity, which is the power system dispatchability and indicates the range of deviation from the predetermined operation point that the system can accommodate. For the 
energy system with multiple generators, the shadow rectangle will be expanded into a polyhedron. Figure 1 shows that power system dispatchability is associated with the TG operation status, ramping limit, and response time. In addition, the power system dispatchability is directly proportional to the limit of the transmission line capacity. The power system dispatchability is affected by various factors. Note that the power system dispatchability can be used to assess the tolerable level of prediction error. In fact, the assessment of power system dispatchability is equivalent to the evaluation of wind energy accommodation. The former calculates the largest regulation range of power system from the perspective of TG, while the latter evaluates the maximum operation interval of power system from the perspective of WT.

As we all know, the dynamic economic dispatch model of power system aims to determine the production plan of generator in the day-ahead and real-time phase. However, wind energy spillage and load demand curtailment often occur in the re-dispatch stage instead of the pre-dispatch stage. The prediction error in the pre-dispatch stage will make the power system deviate from the predetermined operation state in the real-time dispatching stage. If the deviation is less than the power system dispatchability, wind energy spillage and load demand curtailment can be avoided. On the contrary, if the deviation is greater than the power system dispatchability, it is necessary to load demand shedding or wind energy spillage to retain the energy balance [18].

\section{ASSESSMENT OF WIND POWER ACCOMMODATION}

The existing research on wind accommodation is based on the static economic dispatch model of the power system, and the indicator is the range of wind power that the system can be accommodated in a single period. This evaluation model is optimistic as the security constraints of the power system with time coupling characteristics (i.e., ramping constraints) are ignored. This paper proposes an evaluation framework of wind energy accommodation based on the theory of dynamic economic dispatch, and the metric indicator is the wind energy envelope that the power system can be accommodated.

The assessment of wind energy accommodation is divided into three steps as the power system dispatchability is limited by the operation status. First, the pre-dispatch scheme is obtained by solving the SCUC problem, and which is defined as the initial operation state of the electric power system. Second, the re-dispatch scheme is calculated through adaptive robust optimization technology. Third, the accommodation envelope of wind power is acquired through the pre-dispatch scheme and re-dispatch scheme.

\section{A. Day-Ahead Dispatch Model}

As the wind energy accommodation and power system dispatchability depend on the operation state, it is necessary to determine the basic operation point of the power system, which is obtained by solving the SCUC model according to the prediction data of wind out power and load demand. Equation (5) represents minimizing the start-up/shut-down costs and fuel costs of TG

$$
\min c^{\prime} x+b^{\prime} y
$$

The binary variable $x$ includes unit commitment, startup state, and shut-down state of TG in the each period. The decision variable $y$ indicates the dispatching decisions in each period, such as generator production power, transmission line power, and phase angle. $c$ ' represents the shut-down/start-up cost parameter of $\mathrm{TG}, b$ ' represents the production cost parameter of the TG.

Equation (6) represents minimum on/off time limits, and the logical constraints of the start-up/shut-down actions

$$
A x \leq B \text {. }
$$

Equation (7) represents the constraints of injection power balance, line transmission power, and phase angle of each bus

$$
C y \leq D \text {. }
$$

Equation (8) represents the constraint containing binary and continuous variables, including production power constraints and ramping constraints

$$
E x+F y \leq G .
$$

Equation (9) represents wind production power constraints

$$
I_{u} y=\bar{H} \text {. }
$$

Note that (5)-(9) are a classical mixed-integer liner programming (MILP), which can be directly processed by CPLEX solver.

\section{B. Wind Energy Accommodation Evaluation Model}

Based on the day-ahead scheme, the evaluation model of the largest deviation of wind production power that the power system can be accommodated is proposed. The envelope band of wind energy accommodation is obtained by solving a two-stage robust optimization problem, which can provide warning signals of wind power leakage and load shedding in electric power system operation. The assessment of wind accommodation is written as follows

$$
\min \sum_{t=1}^{T} \sum_{w=1}^{N_{w}} \sum_{d=1}^{N_{d}}\left\{\left(\underline{p}_{w, t}-\bar{p}_{w, t}\right)+\max \left(\Delta p_{w, t}^{r}+\Delta p_{d, t}^{r}\right)\right\}
$$

where $T$ represents time period set, $N_{w}$ and $N_{d}$ represent WT and load node set, respectively, $\underline{p}_{w, t}$ and $\bar{p}_{w, t}$ represent the left and right ends of the largest production interval of WT that power system can be accommodated, respectively, $\Delta p_{w, t}^{r}$ and $\Delta p_{d, t}^{r}$ represent wind curtailment and load shedding, respectively, $p_{w i, t}^{r}$ represents the output power of WT.

$\mathbb{U}$ represents the uncertainty set of wind power output

$$
\mathbb{U}=\left\{\begin{array}{r}
\left(\underline{p}_{w, t}, \bar{p}_{w, t}\right) \mid 0 \leq \underline{p}_{w, t} \leq p_{w, t}^{*} \leq \bar{p}_{w, t} \leq p_{w, \max }, \\
p_{w, t}^{*}-\underline{p}_{w, t}=\bar{p}_{w, t}-p_{w, t}^{*}, \forall t \in T,
\end{array}\right.
$$

where $p_{w, t}^{*}$ represents the predicted power of WT. $p_{w, \max }$ represents the installed capacity of the WT.

The envelope band of wind accommodation is set to be 
symmetrical about the prediction output as the prediction error may be positive or negative

$$
p_{g, t}^{r}+p_{w, t}^{r}-\Delta p_{w, t}^{r}+p_{l, t}^{r}=p_{d, t}^{b}-\Delta p_{d, t}^{r}
$$

where $p_{g, t}^{r}$ and $p_{d, t}^{b}$ represent output power of TG and load demand, respectively, $p_{l, t}^{r}$ represents the transmission flow of the line

$$
p_{g, \min } x_{g, t} \leq p_{g, t}^{r} \leq p_{g, \max } x_{g, t},
$$

where $p_{g, \min }$ and $p_{g, \max }$ represent the lower and upper bounds of the production power of TG, respectively, $x_{g, t}$ indicates unit commitment status of TG, which has been obtained through solving the SCUC problem

$$
p_{g, t}^{r}-p_{g, t-1}^{r} \leq x_{g, t-1} r_{g}^{u} \Delta t+\left(x_{g, t}-x_{g, t-1}\right) p_{g, \min },
$$

where $r_{g}^{u}$ represents the ramping-up constraints of TG, $\Delta t$ indicates a dispatch period (i.e., $\Delta t=1 \mathrm{~h}$ )

$$
p_{g, t-1}^{r}-p_{g, t}^{r} \leq x_{g, t-1} r_{g}^{d} \Delta t+\left(x_{g, t}-x_{g, t-1}\right) p_{g, \min },
$$

where $r_{g i}^{d}$ represents the ramping-down constraints of TG

$$
-r_{g}^{d} \Delta t_{a} x_{g, t} \leq p_{g, t}^{r}-p_{g, t}^{b} \leq r_{g}^{u} \Delta t_{a} x_{g, t},
$$

where $\Delta t_{a}$ is response time, $p_{g, t}^{b}$ is the pre-dispatch power of TG:

$$
\left\{\begin{array}{l}
p_{l, t}^{r}=B_{i j}\left(\theta_{i, t}^{r}-\theta_{j, t}^{r}\right), \\
\left|p_{l, t}^{r}\right| \leq p_{l, \max },
\end{array}\right.
$$

where $B_{i j}$ represents susceptance of transmission lines, $\theta_{i, t}^{r}$ and $\theta_{j, t}^{r}$ represent phase angle of bus $i$ and $j$, respectively, $p_{l, \max }$ represents maximum transmission power

$$
\theta_{i, \min } \leq \theta_{i, t}^{r} \leq \theta_{i, \max }
$$

where $\theta_{i, \min }$ and $\theta_{i, \max }$ represent the lower and upper bounds of the phase angle of bus $i$, respectively:

$$
\left\{\begin{array}{l}
0 \leq \Delta p_{w, t}^{r} \leq p_{w, t}^{r}, \\
\sum_{t=1}^{T} \sum_{w=1}^{N_{w}} \Delta p_{w, t}^{r} \leq \beta_{w} \sum_{t=1}^{T} \sum_{w=1}^{N_{w}} \Delta p_{w, t}^{r} .
\end{array}\right.
$$

Equation (18) represents the wind curtailment constraints, and $\beta_{w}$ is the predetermined wind curtailment ratio:

$$
\left\{\begin{array}{l}
0 \leq \Delta p_{d, t}^{r} \leq p_{d, t}^{b}, \\
\sum_{t=1}^{T} \sum_{d=1}^{N_{d}} \Delta p_{d, t}^{r} \leq \beta_{d} \sum_{t=1}^{T} \sum_{d=1}^{N_{d}} p_{d, t}^{b} .
\end{array}\right.
$$

Similarly, $\beta_{d}$ is the predetermined load shedding ratio.

Equations (10)-(20) aim to find the boundary of the wind power to minimize the amount of wind curtailment and load shedding in extreme scenarios. The envelope of wind power accommodation is obtained by solving the nonstandard two-stage robust optimization problem [18]. The model is intractable as the uncertainty set is related to the boundary of the accommodation envelope. Therefore, we need to decouple the uncertainty set and the decision variable by equation (21) and continuous variable [14]

$$
p_{w, t}^{r}=\underline{p}_{w, t} v_{w, t}+\left(1-v_{w, t}\right) \bar{p}_{w, t}, v_{w, t} \in[0,1] .
$$

where $v$ represents the ratio of the actual output power of the WT to the largest operation range that the power system can accommodate, and which is predetermined the uncertainty set. The optimization aims to seek the bound of the envelope band, such that there always finds a practicable corrective action satisfying the constraints of wind curtailment and load shedding at any predetermined uncertain variables $v$.

\section{SOLUTION MEthodologiES}

A solution methodologies based on Benders decomposition are proposed in this section. We note that the optimization problem represented by (10) has the characteristics of multi-stage decision. The first stage of decision is to determine the maximum and minimum value of the wind power before the realization of uncertainty. The second stage of decision is to determine the re-dispatch scheme of power system after the realization of uncertainty. If the predetermined wind power interval is too radical, there will be serious wind power leakage and load shedding in the second stage decision due to the limited dispatch-ability of the power system. To this end, the purpose of two-stage decision-making is that there is always a rescheduling scheme satisfying the security operation constraints for any wind power realization within a predetermined interval. According to Benders algorithm, the second stage decision is recasted as MILP when the first stage decision is predetermined:

$$
\begin{gathered}
\max \min \left(\Delta p_{w}^{r}+\Delta p_{d}^{r}\right), \\
J p_{g}^{r}+K p_{w}^{r}+L \Delta p_{w}^{r}+Q \Delta p_{d}^{r} \geq R, \\
S p_{g}^{r}+T p_{g}^{b} \geq V .
\end{gathered}
$$

Equation (23) corresponds to (11)-(15) and (17)-(21). Equation (24) corresponds to (16). The prediction deviation of wind power production is counterweighted by deploying the ramp-up/down capacity of TG, and the generation power of the TG depends on the production power of the WT. The complex sub-problem can be transformed into a single-level optimization through the strong dual and big-M method:

$$
\begin{gathered}
\max \left(R-K p_{w}^{r}\right)^{\prime} \lambda+\left(V-T p_{g}^{b}\right)^{\prime} \varphi, \\
J^{\prime} \lambda+S^{\prime} \varphi \leq 0, \\
L^{\prime} \lambda \leq 1, \\
Q^{\prime} \lambda \leq 1,
\end{gathered}
$$




$$
\begin{gathered}
p_{w}^{r}=\underline{p}_{w} v_{w}+\left(1-v_{w}\right) \bar{p}_{w}, \\
\lambda, \varphi \geq 0, v_{w} \in[0,1],
\end{gathered}
$$

where $\lambda$ and $\varphi$ are Lagrange multipliers corresponding to equations (23) and (24). We note that the problem is still an intractable bilinear optimization as the bilinear term [19]

$$
p_{w}^{r} \lambda=\underline{p}_{w, t} v_{w, t} \lambda+\left(1-v_{w, t}\right) \bar{p}_{w, t} \lambda, \forall t \in T, v_{w, t} \in[0,1]
$$

The results show that the optimal decision of bilinear optimization can be obtained by searching the extreme points of feasible regions [20], which means that the continuous variable $v_{i, t}$ is similar to the binary variables. Disjunctive inequalities are used to linearize nonlinear terms [21]:

$$
\begin{aligned}
& \gamma_{w, t}-\lambda \underline{p}_{w, t}+M_{w}\left(1-v_{w, t}\right) \geq 0, \\
& \gamma_{w, t}-\lambda \underline{p}_{w, t}+M_{w}\left(1-v_{w, t}\right) \leq 0, \\
& \gamma_{w, t}-\lambda \bar{p}_{w, t}+M_{w} v_{w, t} \geq 0 \\
& \gamma_{w, t}-\lambda \bar{p}_{w, t}+M_{w} v_{w, t} \leq 0 .
\end{aligned}
$$

Equation (28) is equivalent to equation (27) by creating a continuous variable $\gamma_{i, t}$ and sufficiently large constant $M_{w}$.

After theoretical derivation, the sub-problem has been rewritten as the tractable MILP problem. Besides, the worst-case scenario and re-dispatch scheme are obtained to solve the mater problem through commercial software if the decision variables of the first stage are predetermined:

$$
\begin{gathered}
\min _{\underline{p}_{w}, \bar{p}_{w}}\left(\underline{p}_{w}-\bar{p}_{w}\right)+\alpha, \\
\alpha \geq\left(R-K p_{w}^{r}\right)^{\prime} \lambda+\left(V-T p_{g}^{b}\right)^{\prime} \varphi, \\
0 \leq \underline{p}_{w} \leq p_{w}^{*} \leq \bar{p}_{w} \leq p_{w, \max },
\end{gathered}
$$

where $a$ represents the auxiliary variable created. Equation (30) represents the constraint generated from the sub-problem that is the optimality cuts [18].

In summary, the envelope of wind power accommodation can be obtained by Benders algorithm alternately solve the master problem and the sub-problem. The outline of Benders decomposition algorithm is as below:

$-\mathrm{S} 1$ : set the $L B=\inf$ and $U B=\sup$, and $k=1$.

- S2: calculate the masterproblem and update the $L B$.

- S3: calculate the sub-problem to worst-case scenario and dual variables and update the $U B$.

- S4: stop the calculation if the convergence criterion is true. Otherwise, $k=k+1$, and generate the optimal cut, and go to $\mathrm{S} 2$.

\section{NUMERICAL STUDIES}

Numerical examples based on modified IEEE 39-bus test systems are analyzed. The modified test system contains 46 lines and 10 generators, and the operation characteristic parameters are shown in MATPOWER [22]. The economic parameters of the TG are given in Table I. The minimum production power and ramping capacity are set as $30 \%$ and
$20 \%$ of the installed capacity. The response time is set as $10 \mathrm{~min}$. The wind farms with an installed capacity of $500 \mathrm{MW}, 300 \mathrm{MW}, 200 \mathrm{MW}, 400 \mathrm{MW}$, and $600 \mathrm{MW}$ are integrated into the modified test system at nodes $3,10,14,16$, and 26 , respectively. The wind farm power production and load demand profiles are given in Fig. 2. The simulation program written by YALMIP and CPLEX was run in MATLAB 2018, and the average time of each calculation is about 300 seconds.

TABLE I. THERMAL UNIT PARAMETERS.

\begin{tabular}{|c|c|c|c|c|c|c|}
\hline Node & $\begin{array}{c}\text { Cstar } \\
(\mathbf{\$})\end{array}$ & $\begin{array}{c}\text { Cshut } \\
(\mathbf{\$})\end{array}$ & $\begin{array}{c}\text { Ton } \\
(\mathbf{h})\end{array}$ & $\begin{array}{c}\text { Toff } \\
(\mathbf{h})\end{array}$ & $\begin{array}{c}\text { Fuel Cost } \\
(\mathbf{\$} / \mathbf{M W h})\end{array}$ & $\begin{array}{c}\text { Pmax } \\
(\mathbf{M W})\end{array}$ \\
\hline 30 & 410 & 320 & 2 & 2 & 40 & 1040 \\
\hline 31 & 460 & 330 & 3 & 1 & 50 & 646 \\
\hline 32 & 450 & 280 & 1 & 1 & 60 & 725 \\
\hline 33 & 400 & 350 & 2 & 1 & 35 & 652 \\
\hline 34 & 400 & 300 & 3 & 2 & 45 & 508 \\
\hline 35 & 330 & 220 & 4 & 4 & 55 & 687 \\
\hline 36 & 300 & 260 & 2 & 1 & 75 & 580 \\
\hline 37 & 150 & 100 & 2 & 1 & 90 & 564 \\
\hline 38 & 180 & 120 & 2 & 2 & 80 & 865 \\
\hline 39 & 190 & 170 & 3 & 3 & 85 & 1100 \\
\hline
\end{tabular}

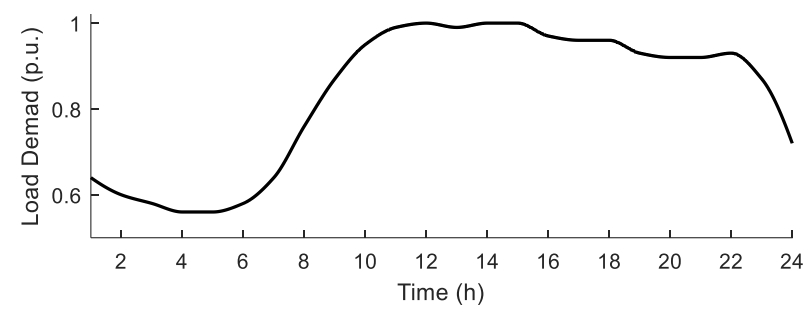

(a)

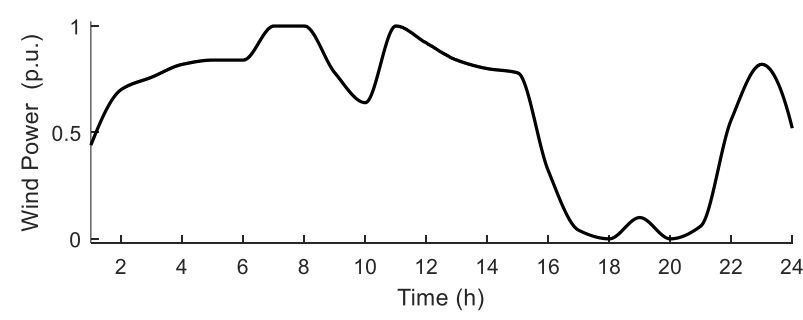

(b)

Fig. 2. (a) The load demand and (b) wind power production profiles.

\section{A. Result of SCUC}

To begin with, the SCUC model based on the predicted data is solved to get the initial operation state of the power system. The regulation capacity of TG is abundant, which makes the predicted power of wind energy can be fully accommodated. Wind power production is $29164 \mathrm{MWh}$, TG generation is $95295 \mathrm{MWh}$, and load demand is $124460 \mathrm{MWh}$. The total operating cost is 4904574 \$. The scheduling for all generators is given in Fig. 3.

The data in the Fig. 3 show that the TG has start-up and shut-down actions, in which the expensive unit is shut down to reduce the generation cost of the power system. When wind power increases and loads demand decreases (i.e., at 22:00), TG needs to contribute ramp-down capacity to prevent wind energy curtailment. Besides, when wind power decreases and load demand increases (i.e., at 11:00), TG needs to contribute ramp-up capacity to prevent load shedding. Results in terms of the number of TG online are shown in Fig. 4. 


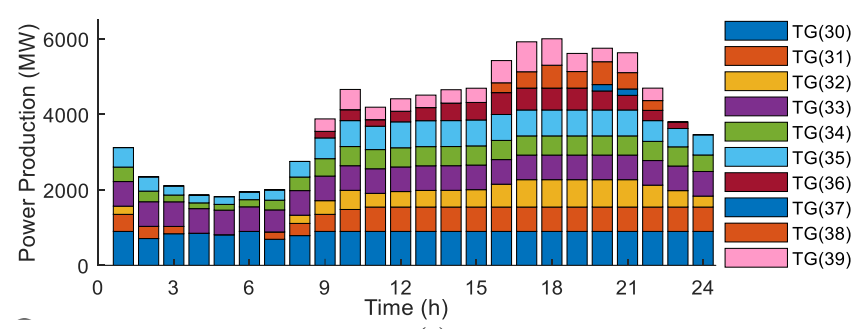

(a)

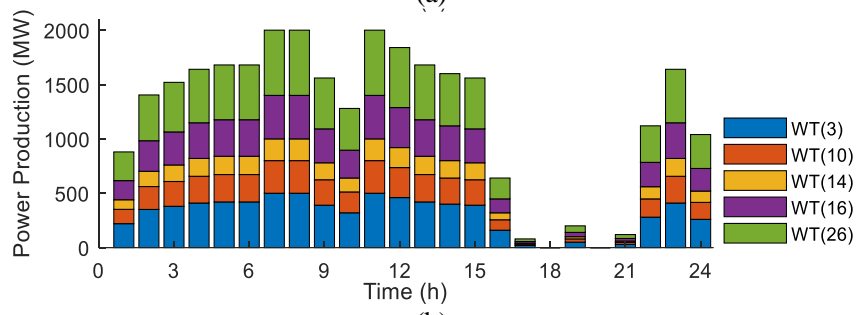

(b)

Fig. 3. Hourly power production for all generators.

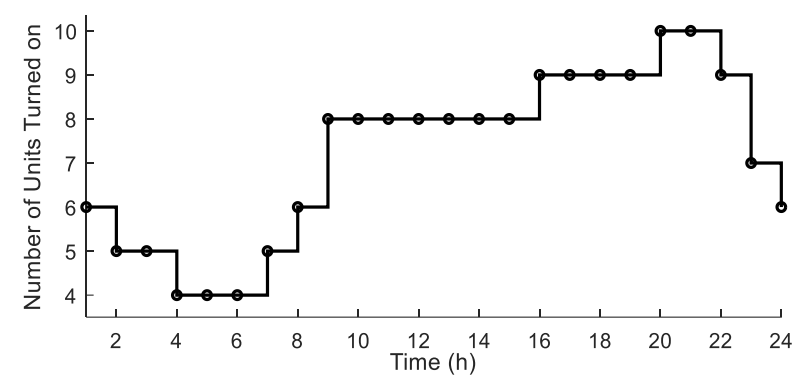

Fig. 4. The number of units turned on.

All TG are involved in the daily dispatch. TG located on nodes $30,33,34$, and 35 are always in the turned-on state, and the other units have start-up and shut-down actions. Due to the limitation of the ramping capacity of TG, the excessive prediction deviation of wind power will lead to wind energy curtailment and load shedding. Applying the framework to assess the largest wind power interval that the power system can accommodate, the results are given in Section V-B.

\section{B. Assessment of Wind Energy Accommodation}

In this section, the output deviation range of all WT nodes is calculated through the solution strategy in Section IV, which is the envelope band of wind power accommodation. To verify the influence of wind energy leakage and load shedding on wind power envelope that electric power system can be accommodated, we designed the following four cases:

- Case 1 is the most conservative $\left(\beta_{w}=0 \%\right.$ and $\beta_{d}=$ $0 \%)$

- Case 2 is load shedding only ( $\beta_{w}=0 \%$ and $\left.\beta_{d}=5 \%\right)$;

- Case 3 is wind power leakage only $\left(\beta_{w}=5 \%\right.$ and $\beta_{d}=$ $0 \%)$

- Case 4 is the most radical $\left(\beta_{w}=5 \%\right.$ and $\beta_{d}=5 \%$ ).

The hourly allowable wind power deviation in different cases is given in Fig. 5.

The data in Fig. 5 shows that there are some time periods with allowable wind power deviation of 0 in all four cases. The results mean that the ability of power system to deal with wind power fluctuations is different in temporal. Wind power continuously ramping-up or ramping-down will cause wind power leakage and load shedding, which will greatly consume the dispatchability resources of power system. Besides, the mathematical statistics indexes are used to evaluate the allowable deviation of wind power in each period of four cases, and the results are given in Table II.

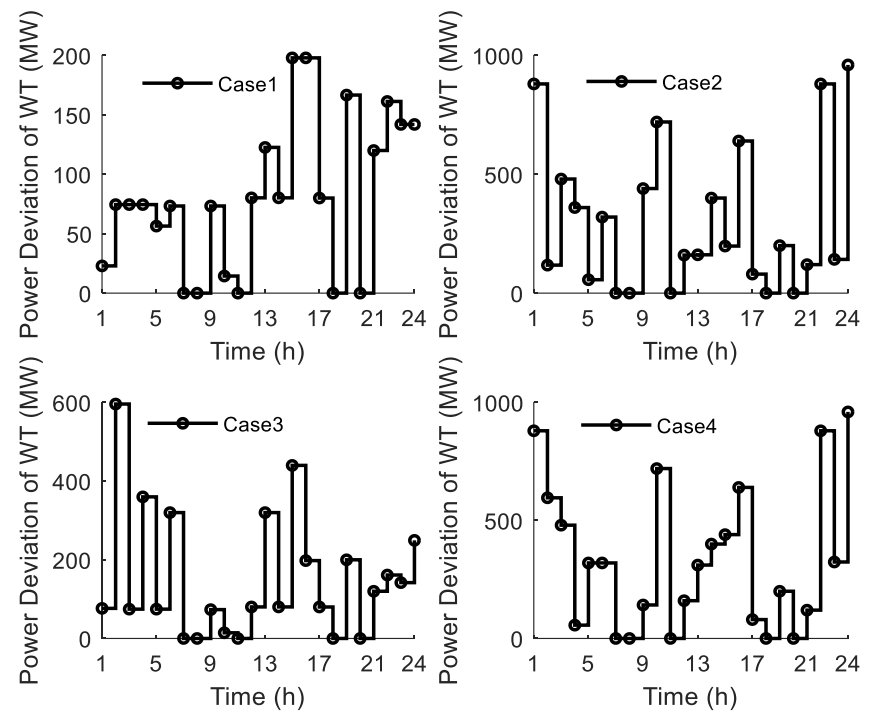

Fig. 5. Wind power deviation in different cases.

TABLE II. STATISTICAL INDEXES OF ALLOWABLE WIND POWER DEVIATION IN EACH PERIOD.

\begin{tabular}{|c|c|c|c|c|}
\hline Index & Case 1 & Case 2 & Case 3 & Case 4 \\
\hline Maximum Value & 198 & 960 & 596 & 960 \\
\hline Minimum Value & 0 & 0 & 0 & 0 \\
\hline Average Value & 82 & 305 & 153 & 335 \\
\hline Standard Deviation & 64 & 307 & 157 & 307 \\
\hline
\end{tabular}

Note that the maximum adjusted output of all TG within the response time is $246 \mathrm{MW}$. Therefore, there is wind spillage and load curtailment in Case 2, Case 3, and Case 4. As the load demand is far greater than the installed capacity of WT, the allowable wind power deviation of Case 2 is greater than Case 3. Both wind power leakage and load shedding are allowed in Case 4, which makes the maximum allowable wind power deviation. The envelope band is given in Fig. 6.

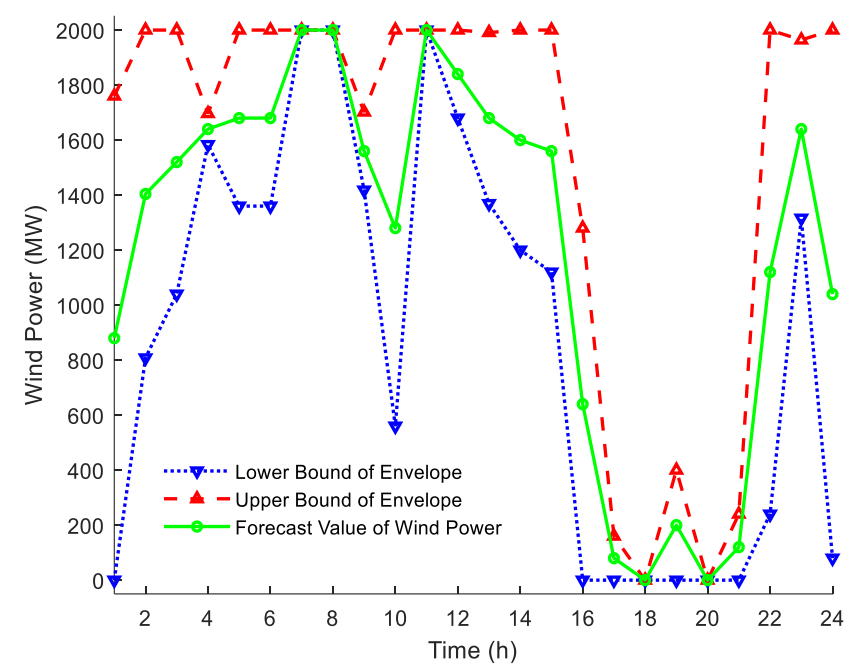

Fig. 6. The envelope band of wind power accommodation in Case 4.

The envelope band in Fig. 6 has such properties: for the realization of arbitrary wind power within the envelope, the amount of wind leakage or load shedding in the re-dispatch stage is acceptable through correcting the pre-dispatch scheme. Once the prediction deviation of wind power exceeds 
the range of wind accommodation envelope, wind leakage or load shedding will run out of predetermined constraints. The envelope band is similar to a metric and can provide early warning information. Furthermore, the hourly wind leakage or load shedding in Case 4 are given in Fig 7(a) and Fig. 7(b).

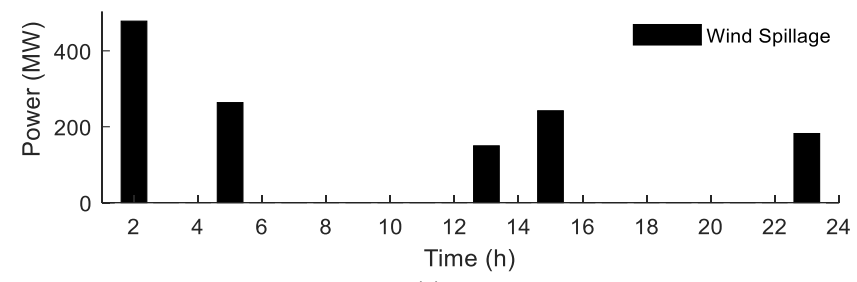

(a)

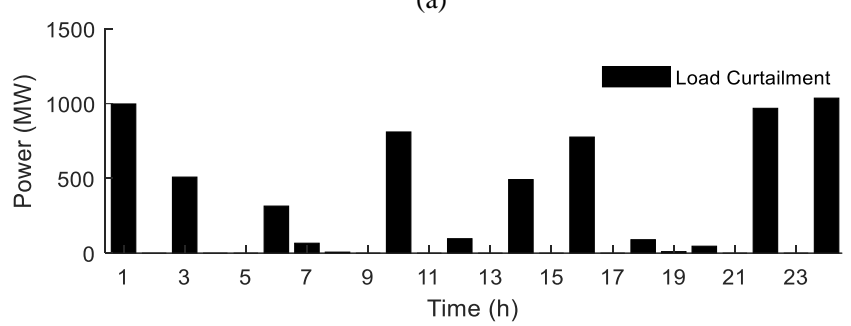

(b)

Fig. 7. (a) Hourly wind energy leakage in Case 4; (b) Hourly load shedding in Case 4.

The total amount of wind spillage and load curtailments are $1316 \mathrm{MWh}$ and $6223 \mathrm{MWh}$, respectively. Wind spillage occurs in $2^{\text {nd }}, 5^{\text {th }}, 13^{\text {th }}, 15^{\text {th }}$, and $23^{\text {th }}$ periods, while the amount of load curtailment is zero. Note that the amount of load curtailment is both zero in $4^{\text {th }}, 9^{\text {th }}, 11^{\text {th }}, 17^{\text {th }}$, and $21^{\text {th }}$ periods. Reasonable wind power leakage and load shedding can significantly improve the ability of the system to withstand net load fluctuations.

\section{CONCLUSIONS}

Based on the pre-dispatch and re-dispatch theory, the dispatchability of the power system is introduced, and the evaluation framework of wind energy accommodation is proposed. The results of numerical studies show that:

1. It is feasible to counterweight the deviation of wind power by correcting the pre-dispatch scheme. The effect of this method is related to the dispatchability resource of the electric power system.

2. Allowing certain wind spillage and load curtailment can greatly improve the ability of the grid to cope with wind power deviation. The results show that the reasonable wind spillage or load curtailment is beneficial to power system scheduling operation.

3. The envelope band of wind power accommodation represents the maximum range of wind power deviation for the power system, which can provide warning information (wind curtailment or load shedding) for decision-makers.

The envelope of wind power accommodation is affected by the electric power system pre-dispatching scheme. Therefore, the problem of co-optimization of the pre-dispatch scheme and envelope band of wind energy accommodation is worthy of attention in the future work. Besides, the deployment of energy storage to improve the envelope band of wind power accommodation is concerned.

\section{CONFLICTS OF INTEREST}

The authors declare that they have no conflicts of interest.

\section{REFERENCES}

[1] T. S. L. V. Ayyarao, "Modified vector controlled DFIG wind energy system based on barrier function adaptive sliding mode control", Prot. Cont. Mod. Power Syst., vol. 4, article No. 4, 2019. DOI: 10.1186/S41601-019-0119-3.

[2] C. Jung, D. Schindler, and J. Laible "National and global wind resource assessment under six wind turbine installation scenarios", Energy Convers. Manage., vol. 156, pp. 403-415, Jan. 2018. DOI: 10.1016/J.ENCONMAN.2017.11.059.

[3] H. Saber, M. Moeini-Aghtaie, M. Ehsan, and M. Fotuhi-Firuzabad "A scenario-based planning framework for energy storage systems with the main goal of mitigating wind curtailment issue", Int. J. Electr. Power Energy Syst., vol. 104, pp. 414-422, Jan. 2019. DOI: 10.1016/J.IJEPES.2018.07.020.

[4] X. Gou, Q. Chen, K. Hu, H. Ma, L. Chen, X. Wang et al., "Optimal planning of capacities and distribution of electric heater and heat storage for reduction of wind power curtailment in power systems", Energy, vol. 160, pp. 763-773, Oct. 2018. DOI: 10.1016/J.ENERGY.2018.07.027.

[5] L. Ye, C. Zhang, H. Xue, J. Li, P. Lu, and Y. Zhao, "Study of assessment on capability of wind power accommodation in regional power grids", Renew. energy, vol. 133, pp. 647-662, Apr. 2019. DOI: 10.1016/J.RENENE.2018.10.042.

[6] E. Lannoye, D. Flynn, and M. O’Malley, "Evaluation of power system flexibility”, IEEE Trans. Power Syst., vol. 27, no. 2, pp. 922-931, 2013. DOI: 10.1109/TPWRS.2011.2177280.

[7] J. Zhao, T. Zheng, and E. Litvinov, "A unified framework for defining and measuring flexibility in power system", IEEE Trans. Power Syst., vol. 31, no. $1, \quad$ pp. 339-347, 2015. DOI: 10.1109/TPWRS.2015.2390038.

[8] B. Mohandes, M. S. E. Moursi, N. Hatziargyriou, and S. E. Khatib, "A review of power system flexibility with high penetration of renewables", IEEE Trans. Power Syst., vol. 34, no. 3, pp. 2457-2460, 2019. DOI: 10.1109/TPWRS.2019.2897727.

[9] H. Nosair and F. Bouffard, "Flexibility envelopes for power system operational planning", IEEE Trans. Sustain. Energy, vol. 6, no. 3, pp. 800-809, 2015. DOI: 10.1109/TSTE.2015.2410760.

[10] W. Wei, F. Liu, and S. Mei, "Real-time dispatchability of bulk power systems with volatile renewable generations", IEEE Trans. Sustain. Energy, vol. 6, no. 3, pp. 738-747, 2015. DOI: 10.1109/TSTE.2015.2413903.

[11] M. Zhou, M. Wang, J. Li, and G. Li, "Multi-area generation-reserve joint dispatch approach considering wind power cross-regional accommodation", CSEE J. of Power and Energy Syst., vol. 3, no. 1, pp. 74-83, 2017. DOI: 10.17775/CSEEJPES.2017.0010.

[12] L. F. Ochoa and G. P. Harrison, "Minimizing energy losses: Optimal accommodation and smart operation of renewable distributed generation", IEEE Trans. Power Syst., vol. 26, no. 1, pp. 198-205, 2011. DOI: 10.1109/TPWRS.2010.2049036.

[13] G. Li, G. Li, and M. Zhou, "Model and application of renewable energy accommodation capacity calculation considering utilization level of inter-provincial tie-line", Prot. Cont. Mod. Power Syst., vol. 4, article No. 1, 2019. DOI: 10.1186/S41601-019-0115-7.

[14] J. Zhao, T. Zheng, and E. Litvinov, "Variable resource dispatch through Do-Not-Exceed limit", IEEE Trans. Power Syst., vol. 30, no. 2, pp. 820-828, 2015. DOI: 10.1109/TPWRS.2014.2333367.

[15] Z. Li, F. Qiu, and J. Wang, "Data-driven real-time power dispatch for maximizing variable renewable generation", Appl. Energy, vol. 170, pp. 304-313, May. 2016. DOI: 10.1016/J.APENERGY.2016.02.125.

[16] Y. Ding, M. Xie, Q. Wu, and J. Østergaard, "Development of energy and reserve pre-dispatch and re-dispatch models for real-time price risk and reliability assessment", IET Gener. Transmiss. Distrib., vol. 8, no. 7, pp. 1338-1345, 2014. DOI: 10.1049/IET-GTD.2013.0822.

[17] A. S. Xavier, F. Qiu, F. Wang, and P. R. Thimmapuram, "Transmission constraint filtering in large-scale security-constrained unit commitment", IEEE Trans. Power Syst., vol. 34, no. 3, pp. 2457-2460, 2019. DOI: 10.1109/TPWRS.2019.2892620.

[18] K. Tian, W. Sun, C. Yang, D. Han, W. Zhang, and P. Xi, "Evaluation method of power system flexibility for renewable energy accommodation", in Proc. of 8th Renewable Power Generation 
Conference, Shanghai, 2019. DOI: 10.1049/cp.2019.0387.

[19] A. G. Nahapetyan, "Bilinear Programming", in Encyclopedia of Optimization. Springer, Boston, MA, 2008.

[20] T. Ding, S. Liu, W. Yuan, Z. Bie, and B. Zeng, "A two-stage robust reactive power optimization considering uncertain wind power integration in active distribution networks", IEEE Trans. Sustain. Energy, vol. 7, no. 1, pp. 301-311, 2016. DOI: 10.1109/TSTE.2015.2494587.
[21] L. Baringo and A. Baringo, "A stochastic adaptive robust optimization approach for the generation and transmission expansion planning", IEEE Trans. Power Syst., vol. 33, no. 1, pp. 792-802, 2018. DOI 10.1109/TPWRS.2017.2713486.

[22] R. Zimmerman, C. Murillo-Sánchez, and R. Thomas, "MATPOWER Steady-state operations, planning, and analysis tools for power systems research and education", IEEE Trans. Power Syst., vol. 26, no. 1, pp. 12-19, 2011. DOI: 10.1109/TPWRS.2010.2051168. 